\title{
New-type Environmental Construction of Nursing Homes Based on the Construction of Aging-friendly Facilities and the Application of Aromatic Plants
}

\author{
Chen Hua*, Mo Jiajie, Cai Miaoqing, Zhang Ruiqi, Zhang Hongping, Liang Rui
}

The Department of Landscape Architecture College of Life Science, Zhaoqing University, Zhaoqing, China

Email address:

chenhuazhp@163.com (Chen Hua)

${ }^{*}$ Corresponding author

\section{To cite this article:}

Chen Hua, Mo Jiajie, Cai Miaoqing, Zhang Ruiqi, Zhang Hongping, Liang Rui. New-type Environmental Construction of Nursing Homes Based on the Construction of Aging-friendly Facilities and the Application of Aromatic Plants. Humanities and Social Sciences.

Vol. 8, No. 1, 2020, pp. 35-44. doi: 10.11648/j.hss.20200801.16

Received: February 6, 2020; Accepted: March 2, 2020; Published: March 10, 2020

\begin{abstract}
The report of the Nineteenth National Congress of the Communist Party of China put forward that we should implement the "Healthy China Strategy" and actively deal with the aging of the population, The problem between the rapid increase in the number of the elderly and their living environment needs to be paid attention to and solved urgently, and creating a new pension environment has become a hot spot. In this study, the methods of ergonomics measurement, investigation and analysis and data statistical analysis were used to obtain the data related to the human body size of the elderly, and a set of reference standards for the size of aging facilities were put forward. Taking Huanggang Sanatorium in Duanzhou District of Zhaoqing City as an example, the indoor of nursing homes environment was designed with the standard design dimensions garden chairs and aromatic plants. In this environment, the relationship between the different concentrations of the fragrant plants and the physical health indicators of the elderly was quantitatively analyzed through the research methods of human physiology. Meanwhile, the outdoor environment of nursing homes with the standard design dimensions display garden facilities was also designed. The study provides an objective data and scientific basis for the construction of green natural recuperation environment and the study of landscape recuperation function in the future.
\end{abstract}

Keywords: Ergonomics, The Garden Facilities, Aging-friendly, Indoor and Outdoor Environment, Recuperation

\section{Introduction}

Nowadays, with the rapid development of economy and the continuous improvement of medical science and technology in China, the life expectancy of Chinese people has increased, leading to population aging in this country. According to the statistics of the Ministry of Civil Affairs of China, the population of elderly people aged 60 and above nationwide reached 212.42 million as of the end of 2015, exceeding $15.5 \%$ of the total population. Based on the international classification for an aging society, China in the $21^{\text {st }}$ century has become one of the aging countries. The report delivered at the 19th National Congress of the Communist Party of China proposed to implement a "Healthy China Strategy" and to actively respond to the aging of the population. This includes establishing a policy system and a social environment to encourage elderly support, filial piety, and respect for the elderly, promoting the combination of professional medical service and social health care, and accelerating the development of the elder care service industry. The issues brought by the sharp increase in the number of elderly people and their living environments need urgent attention and addressing. Creating a new elder-friendly environment has become a heatedly discussed topic.

Ergonomics is a discipline that studies the relationship between humans and their external environment [1-2]. By analyzing people's behaviours and habits, psychological factors, thinking patterns, and the physiological structure of the human body, ergonomics promotes human-friendly designs to fully reflect the care for humans and respect for human nature. Designing to "human scale" refers to the use of the scale of the human body as an indicator in landscape 
architecture to ensure the scale of the external environment, facilities, and psychological proximity is designed for the physical comfort of humans during their activities, rest, or communication. Human scale is not a fixed value in a landscape, but a variable that constantly changes according to the scale of different human bodies or different population groups. The body size of an elderly person is slightly smaller than that of a young or middle-aged person. Such differences may cause huge obstacles in the use of daily facilities. The difference in the body size is more obvious for a disabled elderly person in a wheelchair [3]. At present, the research on the application of ergonomics in human scale design is mainly focused on the furniture design in an indoor environment [4-6]. Jiao Ren studied the development trend of ergonomic design based on seven kinds of furniture [7]. Pengyu Ren reported on furniture design in a residential setting for the elderly, and proposed that due to the subtle changes in the physical dimensions of the aging body of the elderly, furniture should be designed to their physiological and psychological needs [8]. Yan Li conducted a study on the kitchen design in the homes of the elderly in northern cities in China; she analyzed the characteristics and needs of their cooking and dining behaviours, and investigated on the area, storage, furniture, electrical appliances and decoration of the kitchens [9]. In addition, studies were carried out in villas gardens [10], urban outdoor furniture [11], campus landscapes [12], architectural design [13-14], and public spaces within housing [15-16] estates to investigate the application of ergonomics-based design. However, no research or report has been published on the application of ergonomics in an indoor or outdoor nursing environment for the elderly.

Aromatic plants release volatile organic compounds (VOCs) in the environment and act on the human body through human's sense of smell. At present, some researchers at home and abroad mainly use essential oils [17-18] or forests for recreation [19-20] to study the effects of volatile organic compounds of plants on human health. Xiang Gao and Lei Yao put people with hypertension in an outdoor area of aromatic plants such as lavendula officinalis, origanum vulgare, Pelargonium graveolens L'Herit., and Ocimum basilicum, where they breathed in the natural aroma. The systolic and diastolic blood pressures of the subjects were significantly reduced [21]. Hexian Jin measured human physiological indicators before and after her subjects smelled fragrance in an outdoor garden and an indoor laboratory, and conducted a questionnaire survey. She concluded that the plum aroma can result in the decrease of the systolic and diastolic blood pressures, heart rate, and electromyogram of the subjects and achieve the effect of relaxing people's mind [22]. The research of Yan Gao showed that an environment of plants can reduce the systolic pressure, diastolic pressure, and heart rate of humans to a certain extent and help the body and mind to become calm, relaxed, and stress-relieved [23]. Qi $\mathrm{Lu}$ et al. measured the changes in blood pressure, heart rate, and respiratory rate of normal health subjects and borderline hypertensive subjects before and after they breathed Narcissus tazetta L. var. chinensis Roem. aromas. They found that the measured physiological indicators decreased significantly [24]. The study by Jianjun Liao et al. has shown that VOCs in plants had a strong effect on disinfection and skin permeability and a moderate stimulating effect once the VOCs were absorbed through human skin, mucous membranes, or mucous membrane lining the respiratory tract, thereby enhancing human immunity [25]. Reports show that in a forest with $95 \%$ of the tree layer being Indonesian cinnamon, the physical comfort level of that environment is classified as "very comfortable", and it has positive effect on human health [26]. The above research outcomes show that the external environment with aromatic plants has a soothing effect on the mood and a positive impact on both physical and mental health. At present, there have been no reports or studies on the placing of suitable aromatic plants in an elderly environment and its impact.

This study investigated the dimensions of elder-friendly facilities in indoor and outdoor environment for the elderly population based on a large sample size. 7 places with many elderly people were selected for field surveys. 100 local elderly people were invited to complete a questionnaire, and their ergonomic data were collected. This study measured, recorded, and analyzed 10 indicators of the human body, namely the height, sitting height, sitting knee height, sitting hip width, sitting eye height, sitting elbow height, sitting depth, hip-knee distance, shoulder width, and extended arm length. An ergonomic research approach was used based on the acquired values of the above indicators to propose a set of standard dimensions for the design of garden facilities (such as garden chairs, bulletin boards, wash basins, and balustrades), providing objective basic data for elder-friendly facility development. Afterwards, at Huanggang Nursing Home in Duanzhou District, Zhaoqing, a new indoor nursing environment for the elderly was created with garden chairs meeting the designed dimension standards and aromatic plants according to this set of dimension standards for elder-friendly facilities. A quantitative analysis was carried out using an ergonomics-based method in this setting to determine the correlation between the VOC concentration of Hedychium coronarium and the physiological indicators of the elderly people. An outdoor nursing environment with display garden facilities meeting the designed dimension standards was also designed.

\section{Method}

\subsection{Experimental Geographic Locations}

The surveys were conducted in seven places, i.e., Zengcheng Retired Home in Guangzhou, Yuexiu Park in Guangzhou, Lianhuashan Park in Shenzhen, Paifang Park in Zhaoqing, Chengdong Park in Zhaoqing, Liusha People's Park in Puning, and Huanggang Nursing Home in Duanzhou District of Zhaoqing.

\subsection{Experimental Methods}

\subsubsection{Ergonomic Measurement Method}

The study directly measured the body size of the test subject. 
The Feida brand $50 \mathrm{~m}$ tape measure was used to measure indicators of the human body. The study selected 10 indicators of the human body, i.e., height $(\mathrm{H})$, sitting height $(\mathrm{SH})$, sitting knee height (SKH), sitting hip width (SHW), sitting eye height (SEYH), sitting elbow height (SEH), sitting depth (SD), hip-knee distance (HKD), shoulder width (SW), and extended arm length (EAL).

\subsubsection{Survey Analysis Method}

The study selected Guangzhou, Shenzhen, Zhaoqing and Puning in Guangdong Province to conduct field surveys and investigations, and selected females over 55 years old and males over 60 years old for the questionnaire survey in accordance with China's current legal retirement age. The study collected and analyzed human body data to summarize the typical human body scale of the elderly population.

\subsubsection{Statistical Analysis Method}

The study used SPSS19.0 statistical software to perform statistics and analysis on data.

\subsubsection{Research Method on Human Physiology}

The experimental subjects were 30 elderly people randomly selected from Huanggang Nursing Home in Duanzhou District of Zhaoqing. Among them, the proportions of males and females were equal. The study selected four quiet, clean and odorless enclosed rooms in the nursing home with an indoor height of 3.5 meters and an area of 10 square meters as the place for the indoor experiment. Given that the VOCs of Hedychium coronarium are mainly emitted by their flowers and the mass of flowers is positively correlated with the concentration of VOCs in them, three elder-friendly spaces with different VOC concentrations of Hedychium coronarium and standard garden chairs were created in accordance with different masses of the Hedychium coronarium flowers. The VOC concentration of the Hedychium coronarium in the three rooms are $0.5 \mathrm{~g} / \mathrm{m}^{3}, 1.0 \mathrm{~g} / \mathrm{m}^{3}$, and $1.5 \mathrm{~g} / \mathrm{m}^{3}$, respectively. The experiments were carried out on Control Group (in a room without Hedychium coronarium), Treatment Group 1 (0.5 $\left.\mathrm{g} / \mathrm{m}^{3}\right)$, Treatment Group $2\left(1.0 \mathrm{~g} / \mathrm{m}^{3}\right)$, and Treatment Group 3 $\left(1.5 \mathrm{~g} / \mathrm{m}^{3}\right)$. Subjects were required to clean their heads 24 hours prior to the test, not to take any drinks with an irritating effect or medicine, and to maintain a healthy diet and schedule. The experiment design referred to Rui Liang's approach [27]. The subjects first sat still for 10 minutes outside the experimental room, and then sat blindfolded for 4 minutes. The experiment kept the sense of smell as the main influencing factor and prevented other external factors from interfering with the subjects. Then an HEN-8611 electronic blood pressure monitor was used to perform blood pressure and pulse measurement on subjects. The BioExplorer software was used to conduct and record the brain wave measurement. Due to different physical capabilities and willingness of cooperation of the elderly subjects, a total of 20 people completed the entire experiment with valid experimental data.

\section{Result}

\subsection{Measurement Data Analysis on Human Scale of the Elderly Based on Ergonomics}

The results recorded in Table 1 shows that the dimension data obtained by the measurement is representative, reliable, and of small error. Either male or female, the elderly people are shorter than the average adult, but the body circumference is larger than that of the average adult. Their hand-movement space is not as large as that of the average adult.

There were differences in the data of scale indicators between males and females. The biggest difference was the body height. The men were $10.6 \mathrm{~cm}$ taller than the women. The smallest difference was hip-knee distance, where the men exceeded the women by $1.7 \mathrm{~cm}$. The data were used to design elder-friendly facilities of suitable sizes while the design of some facilities should be gender-specific.

Table 1. Table of measurement data and error analysis of human scale of the elderly (Unit: $\mathrm{cm})$.

\begin{tabular}{|c|c|c|c|c|c|c|c|c|c|c|}
\hline gender & $\mathbf{H}$ & SH & SKH & SHW & SEYH & SEH & SD & HKD & SW & EAL \\
\hline male & $168.0 \pm 4.8$ & $86.0 \pm 4.8$ & $55.4 \pm 5.7$ & $43.1 \pm 7.0$ & $74.1 \pm 4.0$ & $27.0 \pm 4.1$ & $46.1 \pm 4.4$ & $55.4 \pm 5.1$ & $50.2 \pm 4.1$ & $73.1 \pm 6.2$ \\
\hline female & $157.4 \pm 5.5$ & $79.7 \pm 5.0$ & $52.0 \pm 6.4$ & $40.3 \pm 6.1$ & $68.8 \pm 5.0$ & $24.8 \pm 2.6$ & $44.4 \pm 4.8$ & $51.8 \pm 6.3$ & $46.8 \pm 5.0$ & $66.8 \pm 6.6$ \\
\hline
\end{tabular}

Note: Abbreviation, height (H), sitting height (SH), sitting knee height (SKH), sitting hip width (SHW), sitting eye height (SEYH), sitting elbow height (SEH), sitting depth (SD), hip-knee distance (HKD), shoulder width (SW), and extended arm length (EAL).

\subsection{Reference Standards for Design Dimensions of Elder-friendly Facilities}

There are different types of garden facilities such as leisure, display, service, and decoration.

\subsubsection{Reference Dimensions of Leisure Garden Facilities}

Leisure garden facilities mainly include garden tables, garden stools, and garden chairs. Among them, the dimensions related to the design of the garden chair include sitting height, chair surface height, armrest height, and backrest length. There was no significant difference in the data between the men and the women. The dimensions of the garden chair design do not have to be gender specific.

Chair height: It can be seen from Table 1 that the knee height of the elderly men in a sitting position was $55.4 \pm 5.7$ $\mathrm{cm}$, and the knee height of the women in a sitting position was $52.0 \pm 6.4 \mathrm{~cm}$. The average knee height value was $53.7 \pm 6.0$ $\mathrm{cm}$. Reference was drawn upon the sitting thigh depth (male: $15.0 \mathrm{~cm}$; female: $14.3 \mathrm{~cm}$ ) and popliteal height (male: $40.9 \mathrm{~cm}$; female: $37.9 \mathrm{~cm}$ ) of average people [28]. Therefore, the height of a suitable elder-friendly chair was set as $38.0-40.1 \mathrm{~cm}$.

Chair surface width: It can be seen from Table 1 that the sitting depth and hip-knee distance of the elderly men were $46.1 \pm 4.4 \mathrm{~cm}$ and $55.4 \pm 5.1 \mathrm{~cm}$, respectively. The sitting 
depth and hip-knee distance of the women were $44.4 \pm 4.86$ $\mathrm{cm}$ and $51.8 \pm 6.3 \mathrm{~cm}$, respectively. The average values were $45.3 \pm 4.6 \mathrm{~cm}$ and $53.6 \pm 5.7 \mathrm{~cm}$, respectively. Therefore, the width of a suitable elder-friendly chair was set as 43.7-46.9 $\mathrm{cm}$.

Armrest height: It can be seen from Table 1 that the elbow height of the elderly men in a sitting posture was $27.0 \pm 4.1 \mathrm{~cm}$, and that of the women was $24.8 \pm 2.6 \mathrm{~cm}$. The average value was $25.9 \pm 3.4 \mathrm{~cm}$. Therefore, the suitable elder-friendly armrest height was set as $24.6-27.3 \mathrm{~cm}$.

Length of the chair surface (single): The length is related to the width between the elbows and the width of the hips in a sitting position. According to Table 1, the width of the elbows between the elbows and the hips for the elderly men in a sitting position were $50.2 \pm 4.1 \mathrm{~cm}$ and $43.1 \pm 7.0 \mathrm{~cm}$, respectively. The values for the women were $46.8 \pm 5.0 \mathrm{~cm}$ and $40.3 \pm 6.1 \mathrm{~cm}$, respectively. The average values were 48.5 $\pm 4.5 \mathrm{~cm}$ and $41.7 \pm 6.5 \mathrm{~cm}$ respectively. Therefore, the length of the chair surface of an elder-friendly single chair was set as $48.0-50.0 \mathrm{~cm}$.

Backrest length: The physical condition of the elderly is not as good as that of the average adult. Their neck muscles are more prone to fatigue. Therefore, the length of the backrest is related to sitting height and sitting eye height. It can be seen from Table 1 that the sitting height and sitting eye height of the elderly men were $86.0 \pm 4.8 \mathrm{~cm}$ and $74.1 \pm 4.0 \mathrm{~cm}$, respectively, and those of the elderly women were $79.7 \pm 5.0$ $\mathrm{cm}$ and $68.8 \pm 5.0 \mathrm{~cm}$, respectively. The average values were $82.8 \pm 4.9 \mathrm{~cm}$ and $71.5 \pm 4.5 \mathrm{~cm}$, respectively. The length of the backrest was set as $67.0-75.0 \mathrm{~cm}$.

The elder-friendly garden chair designed according to the standard dimensions of this study is shown in Figure 1. The comparison with the dimensions of a general garden chair [29] is presented in Table 2. There was a certain degree of difference between the two sets of data.

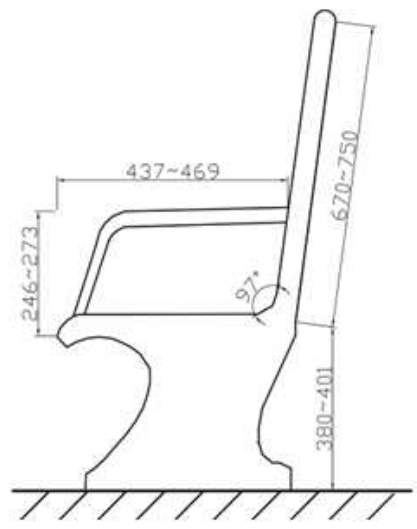

a Side elevation

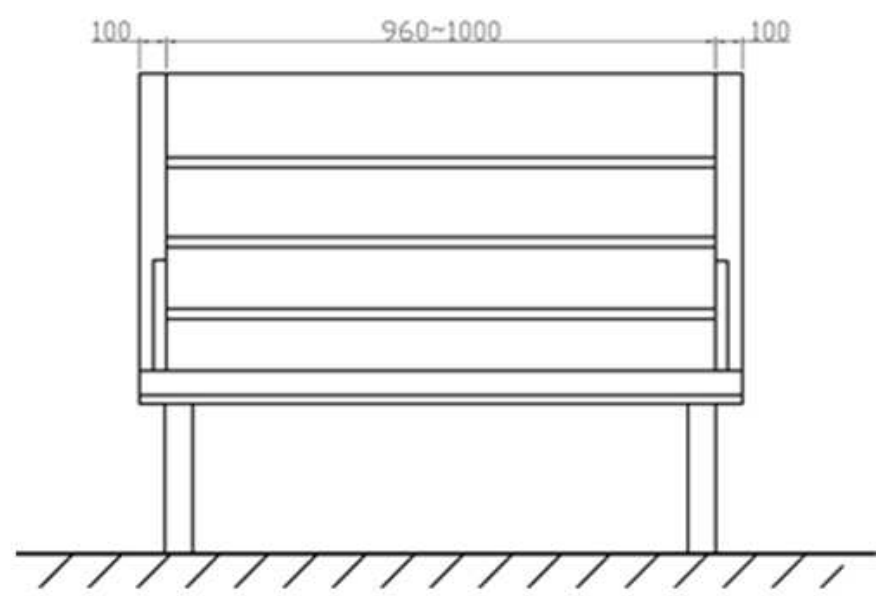

b Front elevation

Figure 1. Garden chair design dimensions of elder-friendly facilities.

Table 2. Comparison table of general size and elder-friendly size of garden chair.

\begin{tabular}{lll}
\hline Name & $\begin{array}{l}\text { general size / } \\
\text { cm }\end{array}$ & $\begin{array}{l}\text { elder-friendly } \\
\text { size / cm }\end{array}$ \\
\hline Chair height & $39-45$ & $38.0-40.1$ \\
Length of the chair surface (single) & $43-45$ & $48.0-50.0$ \\
Chair surface width & $\geq 46$ & $43.7-46.9$ \\
Armrest height & $20-25$ & $24.6-27.3$ \\
Backrest length & $45-50$ & $67.0-75.0$ \\
\hline
\end{tabular}

\subsubsection{Reference Dimensions for Display Garden Facilities}

The design of an elder-friendly bulletin board is mainly for two types of elderly users, i.e., those who can walk freely and those who require a wheelchair for mobility [30].

The bulletin board for the elderly who can walk freely

As presented in Table 1, the height of the elderly men was $168.0 \pm 4.8 \mathrm{~cm}$, and the height of the elderly women was 157.4 $\pm 5.5 \mathrm{~cm}$. The average height was $162.7 \pm 5.1 \mathrm{~cm}$. Since the difference in body height between men and women was greater than $10 \mathrm{~cm}$, the design of an elder-friendly bulletin board must be designed in a gender-specific way. The neck of the elderly is not as flexible as that of an average adult, and the vision of their eyes is not as good as that of the young. The top heights of men's boards and women's boards were set as 182.8 $\mathrm{cm}$ and $172.9 \mathrm{~cm}$, respectively. The bottom heights were set as $92.8 \mathrm{~cm}$ and $82.9 \mathrm{~cm}$, respectively, based on the fact that the general height of an ordinary bulletin board is $90 \mathrm{~cm}$.

The bulletin board for the elderly who require a wheelchair for mobility

Elderly people who require a wheelchair for mobility usually read the bulletin board while sitting, so the height of the bulletin board is designed based on the measured eye height in a sitting position. As presented in Table 1, the average sitting eye height of the elderly was $71.5 \pm 4.5 \mathrm{~cm}$. There was very little difference in this value between men and women. The height of an ordinary wheelchair is $50 \mathrm{~cm}$. Therefore, the top height of the board was set as $161.5 \mathrm{~cm}$ and the bottom height as $71.5 \mathrm{~cm}$.

The design drawing of the different types of the elder-friendly bulletin boards in accordance with the standard dimension data acquired in this study is shown in Figure 2a and Figure $2 \mathrm{~b}$. The comparison with the general dimensions of an ordinary bulletin board is presented in Table 3 . 


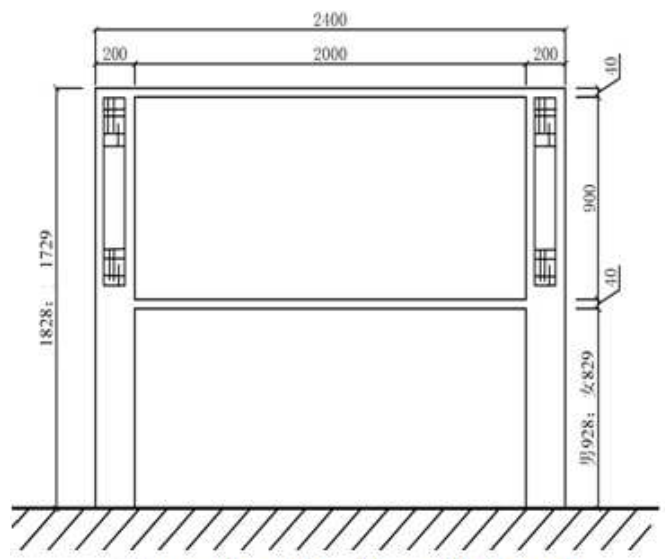

a Front elevation (the elderly who can walk freelv)

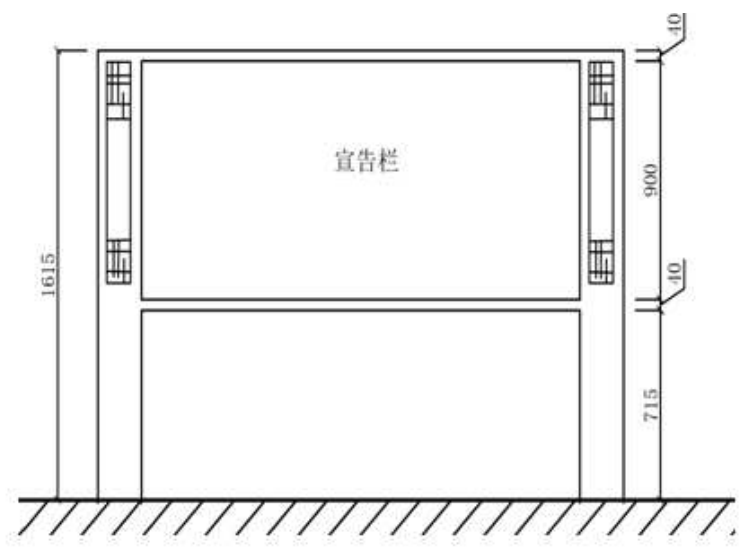

b Front elevation (the elderly who require a wheelchair for mobility)

Figure 2. The bulletin board design dimensions of elder-friendly facilities.

Table 3. Comparison table of general size and elder-friendly size of the bulletin board.

\begin{tabular}{|c|c|c|c|c|}
\hline \multirow{2}{*}{ Name } & \multirow{2}{*}{ general size $/ \mathrm{cm}$} & \multicolumn{2}{|c|}{ Size for the elderly who can walk freely $/ \mathrm{cm}$} & \multirow{2}{*}{$\begin{array}{l}\text { Size for the elderly who require a } \\
\text { wheelchair for mobility /cm }\end{array}$} \\
\hline & & male & female & \\
\hline The top heights & $200-220$ & 182.8 & 172.9 & 161.5 \\
\hline The bottom heights & $115-120$ & 92.8 & 82.9 & 71.5 \\
\hline
\end{tabular}

\subsubsection{Reference Dimensions of Sanitary Garden Facilities}

The wash basin in public service place is designed to the height of the average adult. The height of the basin is generally between $70.0 \mathrm{~cm}$ and $80.0 \mathrm{~cm}$. Due to changes in the body such as less dexterous hands and legs and shorter body height (it can be seen from Table 1 that the average height of the elderly was $162.7 \pm 5.1 \mathrm{~cm}$ ), the height of the wash basin will be slightly lower than average to make it more convenient for

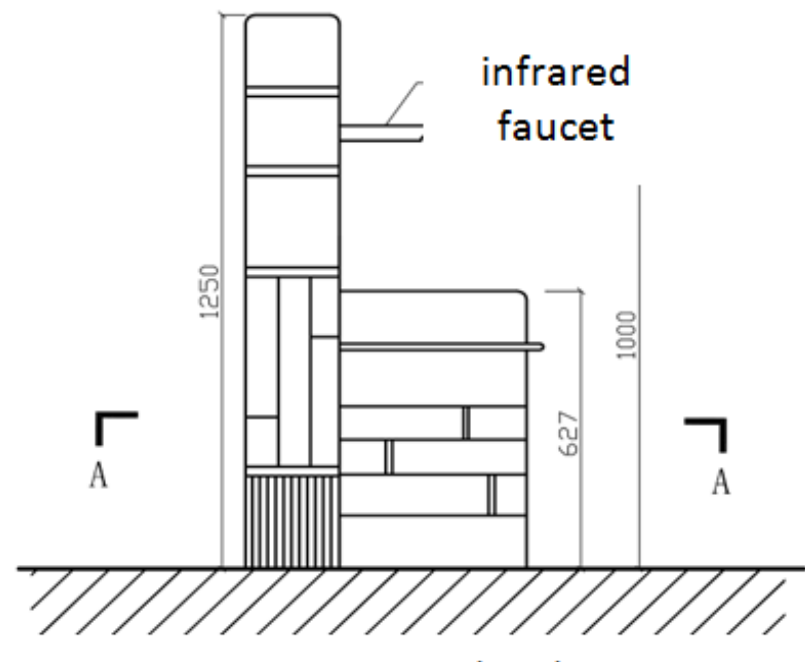

a Front elevation elderly people. The wash basin also uses an automatic faucet with an infrared sensor. The height of the basin was set as 62.7 $\mathrm{cm}$ and that of the infrared faucet was set as $100.0 \mathrm{~cm}$.

The design drawing of the elder-friendly wash basin in accordance with the standard dimension data acquired in this study is shown in Figure 3. The comparison with the general dimensions of an ordinary wash basin [31] is presented in Table 4.

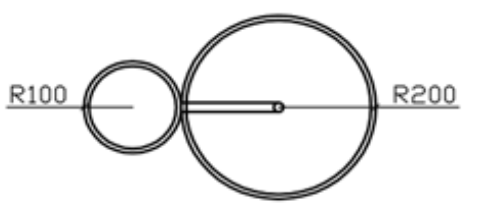

b Plane view

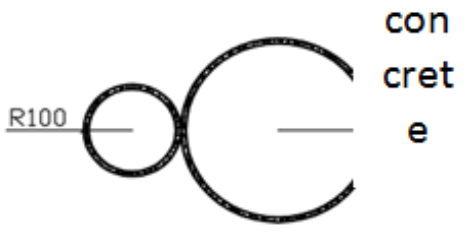

c A-A section

Figure 3. The wash basin design dimensions of elder-friendly facilities.

Table 4. Comparison table of general size and elder-friendly size of the wash basin.

\begin{tabular}{lll}
\hline Name & general size $/ \mathbf{c m}$ & elder-friendly size $/ \mathbf{c m}$ \\
\hline The height of the faucet & $110-115$ & 100.0 \\
The height of the basin & $70-80$ & 62.7 \\
\hline
\end{tabular}

\subsubsection{Reference Dimensions of Protective Garden Facilities}

In gardens, the most frequently used protective service facility among elderly people is the balustrade. Like the bulletin board, there are two types of elder-friendly 
balustrades designed for two types of users. Normally, the height of a balustrade is $110-120 \mathrm{~cm}$. As presented in Table 1, the average height of the elderly was $162.7 \pm 5.1 \mathrm{~cm}$ while the height of an ordinary wheelchair was $50 \mathrm{~cm}$. Since elderly people's hands and legs are not dexterous and they tend to bend over when using balustrades, the heights of the balustrade should be appropriately reduced in the design, which were set as $81.7 \mathrm{~cm}$ and $55.0 \mathrm{~cm}$, respectively.

The design drawing of the elder-friendly balustrade in accordance with the standard dimension data acquired in this study is shown in Figure 4.

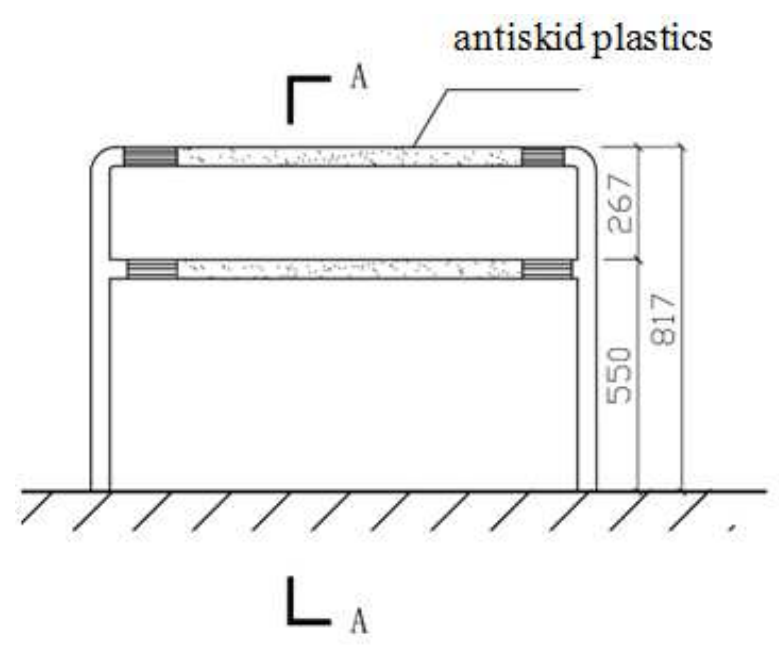

a Front elevation

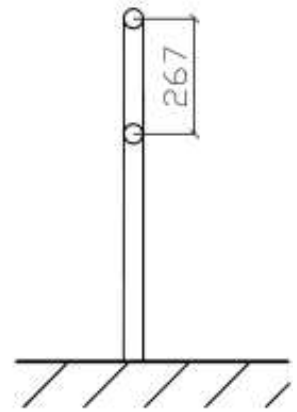

b A-A section

Figure 4. The balustrade design dimensions of elder-friendly facilities.

3.3. Creation of a New Indoor Environment for the Elderly at Huanggang Nursing Home and Analysis on the Health Care Function of Aromatic Plants

\subsubsection{Analysis on the Garden Chair Design}

At Huanggang Nursing Home, a set of standard dimensions for elder-friendly garden chairs was proposed based on this study. The physical comfort of the subjects was improved by adjusting the dimensions of the experimental chairs. At the same time, Hedychium coronarium, a kind of aromatic plant, were placed to create an indoor environment for the elderly (Figure 5) for health care function analysis.

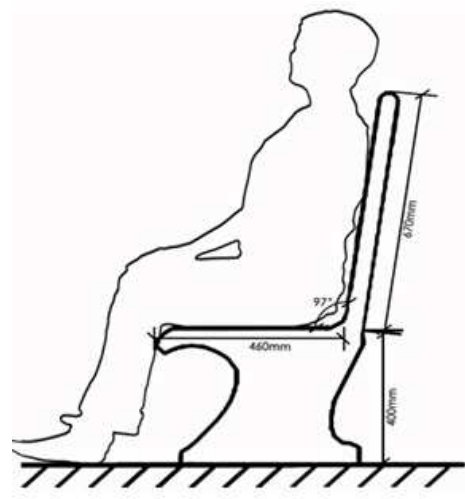

a Side elevation

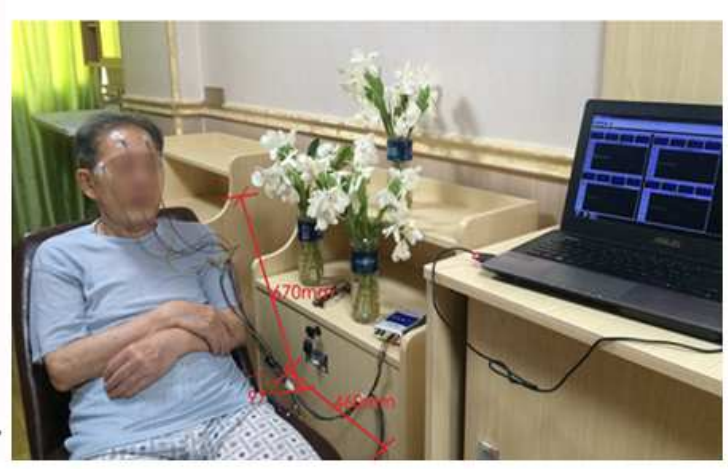

$\mathrm{b}$ Live photos of the experimental

Figure 5. The side elevation design dimensions of garden chair for indoor experiment and Live photos of the experimental.

\subsubsection{Analysis on the Health care Function of Hedychium Coronarium, an Aromatic Plant}

The physiological experimental approach was followed to measure the blood pressure, heart rate, and brain wave of the elderly in the preset elder-friendly indoor environment for the health care function analysis on aromatic plants.

As shown in Figure 6, the systolic blood pressure (Figure 6a), diastolic blood pressure (Figure 6b), and pulse pressure difference (Figure 6c) in the indoor environment of the elderly in Treatment Group 1 (TG1) and Treatment Group 2 (TG2) decreased compared with those from the control group (CK). Among them, Treatment Group 1 demonstrated the best effect. On the other hand, Treatment Group 3 (TG3) showed an increase in the above indicators increased compared with those from the control group. The results in this study showed that when the VOC concentration of Hedychium coronarium in the indoor environment was at $0.5 \mathrm{~g} / \mathrm{m}^{3}$, it exerted the most 
positive effect on the physical health of the elderly. As the concentration increased, the elderly gradually adapted to the aroma of Hedychium coronarium flowers. Their breathing frequency slowed down and heart rate decreased as well. The indoor environment with aromatic plants created in this study demonstrated a certain degree of positive effect on the physical and mental relaxation and the stress relief of the elderly within a certain threshold range of concentration rather than a simple positive correlation.

a

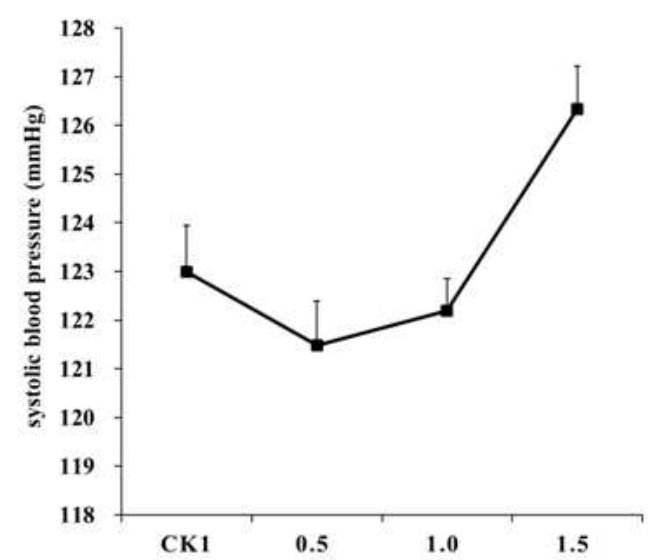

b

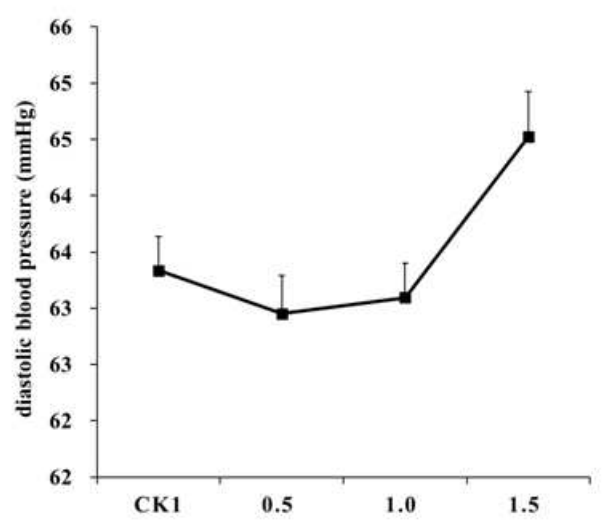

a

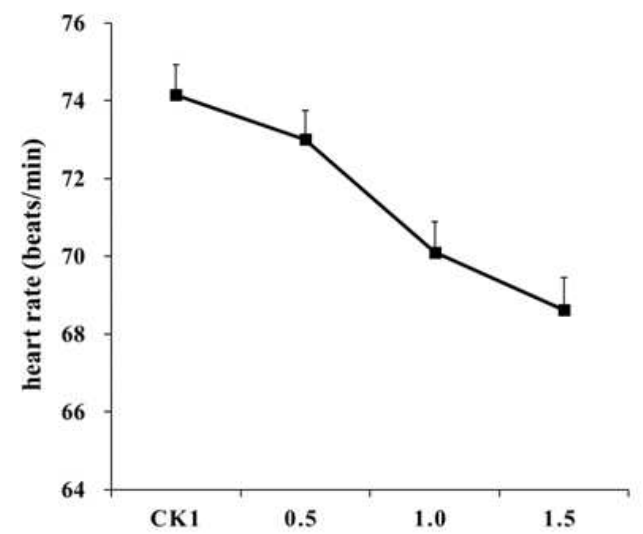

C

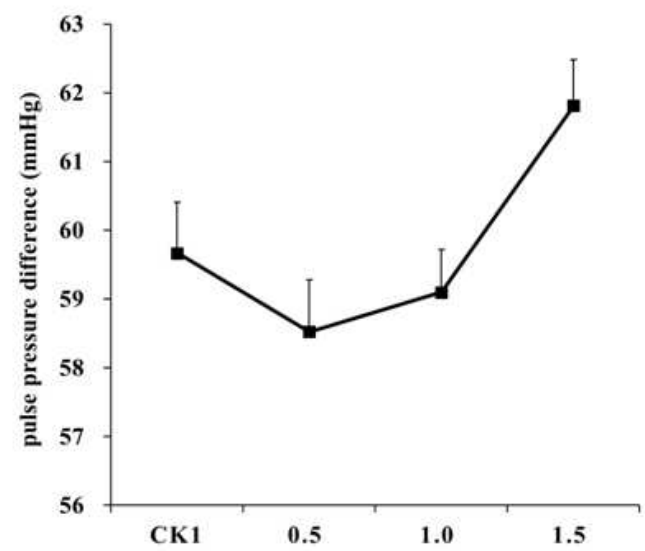

Figure 6. Correlation between different concentrations of $H$. coronarium volatile organic compounds in the indoor environment and the elderly blood pressure. Note: $C K 1,0.5,1.0,1.5$ mean that the VOC concentration of the Hedychium coronarium are $0.0 \mathrm{~g} / \mathrm{m}^{3}, 0.5 \mathrm{~g} / \mathrm{m}^{3}, 1.0 \mathrm{~g} / \mathrm{m}^{3}$, and $1.5 \mathrm{~g} / \mathrm{m}^{3}$.

As shown in Figure 7a, compared with the average heart rate of those from the control group, the average heart rate of the elderly in the treatment groups showed a downward trend as the VOC concentration of Hedychium coronarium flowers in the indoor environment increased. The VOC concentration of the plants showed a negative correlation with the average heart rate of the elderly. As shown in Figure $7 \mathrm{~b}$, the average value of the $\alpha / \beta$ ratio of Treatment Group 2 was the highest among the three groups while the average value of the $\alpha / \beta$ ratio measured by the other two treatment groups was higher than that of the control group. The results show that the indoor environment with different VOC concentrations of Hedychium coronarium flowers had different impacts on the brain waves of the elderly. The effect of Treatment Group 2 was the best, indicating that cerebral cortex activity was at its best status and the human body was in its best physical, mental, and emotional state at that point. The results show that when the concentration of VOCs in Hedychium coronarium flowers in the indoor environment was $1.0 \mathrm{~g} / \mathrm{m}^{3}$, it created the best effect on the physical health of the elderly.

b

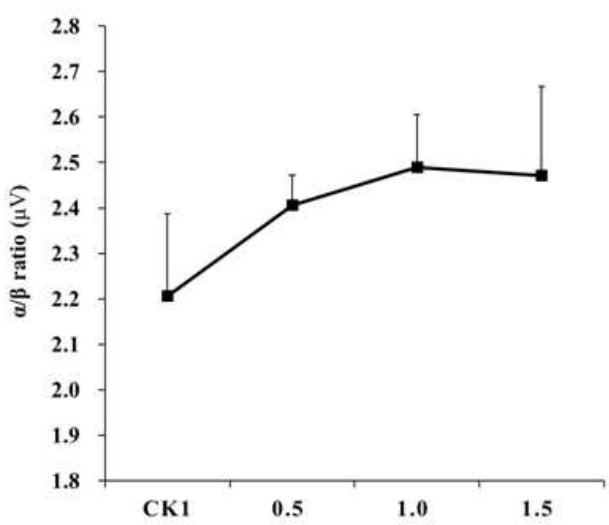

Figure 7. Correlation between different concentrations of $H$. coronarium volatile organic compounds in the indoor environment and the elderly heart rate and brain wave. Note: CK1, 0.5, 1.0, 1.5 mean that the VOC concentration of the Hedychium coronarium are $0.0 \mathrm{~g} / \mathrm{m}^{3}, 0.5 \mathrm{~g} / \mathrm{m}^{3}, 1.0 \mathrm{~g} / \mathrm{m}^{3}$, and $1.5 \mathrm{~g} / \mathrm{m}^{3} .3 .4$. Application Analysis on the Bulletin Board Design 
In the environment of the elderly, some of the bulletin boards are often designed to be too high. The elderly people want to see what is on the bulletin boards but cannot see it. Sometimes they have to stand on their feet. This creates hidden dangers. Therefore, an elder-friendly bulletin board was designed for Huanggang Nursing Home in Duanzhou District of Zhaoqing based on the standard facility dimensions obtained above. As shown in Figure 8, the bulletin board in the category of display garden facilities specially designed for elderly people with different health conditions (healthy elderly people and those who need wheelchairs for mobility) is really based on their needs and more human-friendly and convenient for them to use. In this way, the elderly will be able to obtain knowledge and information on the bulletin boards so that the facilities can play a real part in their life.

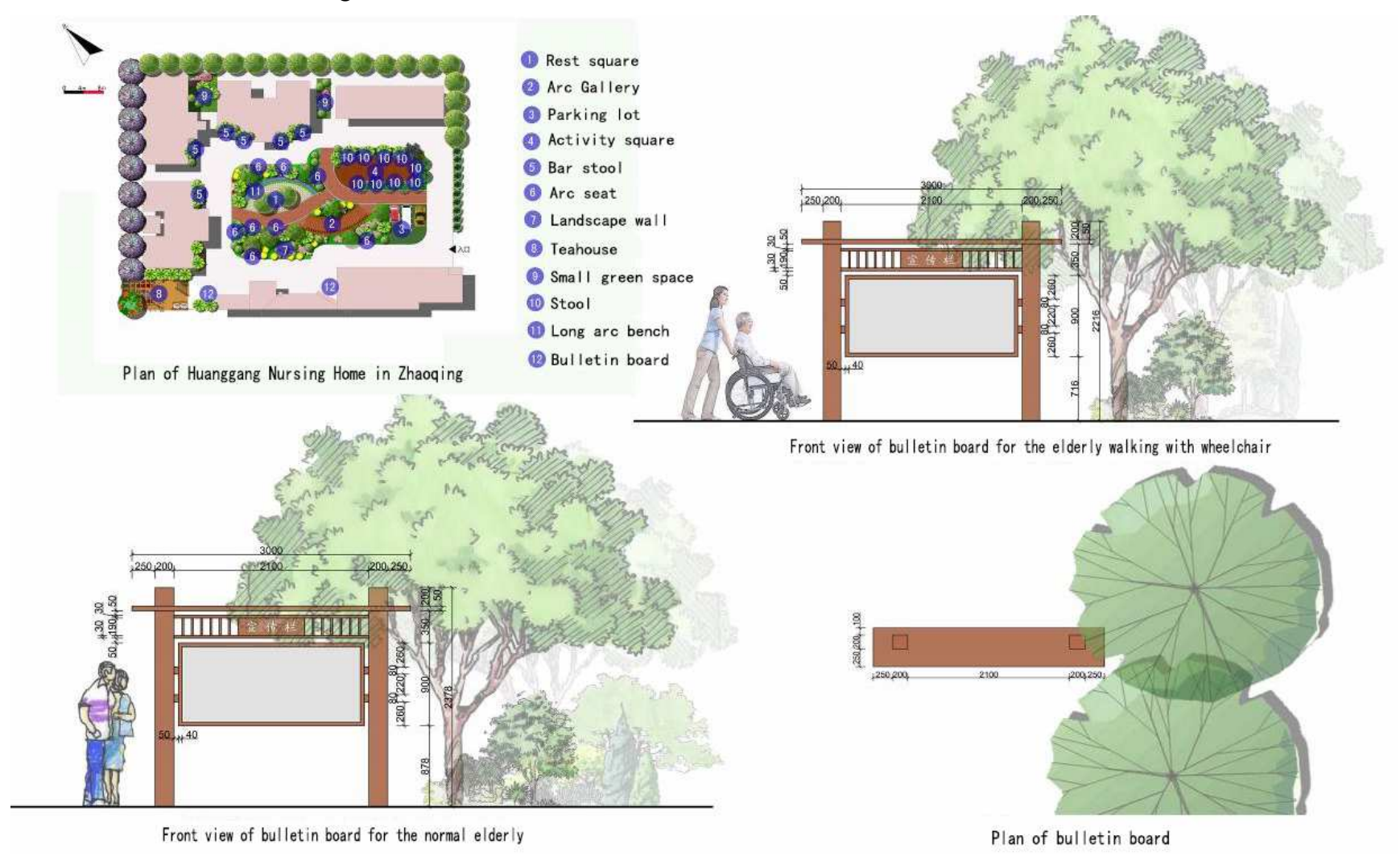

Figure 8. Application example of the bulletin board of elder-friendly facilities.

\section{Discussion}

Systolic blood pressure, diastolic blood pressure, and heart rate among other physiological indicators reflect changes in the autonomic nervous system of the human body. Systolic and diastolic blood pressures and heart rate also reflect the vascular function of the body, which is related to ventricular contractility and peripheral resistance [32]. Studies show that an environment of plants can reduce the systolic pressure, diastolic pressure, and heart rate of humans to a certain extent and help the body and mind to become calm, relaxed, and stress-relieved [23]. Brain waves reflect changes in the indicators of the central nervous system. Brain wave is also a more sensitive indicator that reflects the emotional changes when the sense of smell of the human body is affected by the environment [33]. Cortical activity in the cerebral cortex is measured using the $\alpha / \beta$ ratio [34]. The larger the ratio is, the smoother the human psychological response is. The health care function analysis on Hedychium coronarium in this study shows that when the VOC concentration of Hedychium coronarium in the indoor environment is within a moderate threshold $\left(0.5 \mathrm{~g} / \mathrm{m}^{3}-1.0 \mathrm{~g} / \mathrm{m}^{3}\right)$, it has a positive effect of relaxing the mind and body of the elderly, helps them to concentrate and feel more soothed and relieved emotionally. However, caution is advised when using aromatic plants with a VOC concentration threshold in the indoor environment higher than $1.0 \mathrm{~g} / \mathrm{m}^{3}$, as they have a weaker effect on the emotional relief and the relaxation of mind and body of the elderly, and may even cause negative effects. The research results provided some reference for the exploration of a new nursing environment with elder-friendly facilities combined with aromatic plants.

\section{Conclusion}

This study collected and analyzed the questionnaire surveys and measured data on body dimensions of the elderly people within Guangdong Province. It summarized the characteristics of the human scale of the elderly population, determined the design standards of size dimensions of elder-friendly facilities, 
and provided basic data for the design of environment facilities for the elderly. In order to explore a new type of elderly care environment, this study created an elder-friendly indoor environment with garden chairs meeting the designed dimensions standards and Hedychium coronarium (a kind of aromatic plant) at Huanggang Nursing Home in Duanzhou District of Zhaoqing. Moreover, based on the standard dimensions for facilities, a bulletin board, as a type of display facility in gardens, was designed to suit the outdoor environment for the elderly people.

\section{Acknowledgements}

This work was supported in part by the National Natural Science Foundation of China to Hua Chen (grant No. 31600573), the National College Students' innovation and entrepreneurship project of China to Mo Jiajie (grant No. 201910580012), the Key platform and scientific research project of Guangdong Education Department to Hua Chen (grant No. 2017KTSCX196).

\section{References}

[1] DONG Biao. Application of ergonomics in indoor environment [J]. MING (Attitude), 2019 (7): 164.

[2] XU Yuanyuan, CHEN Zuzhan, LIU Zihui. Research of xiang nan traditional residential house interior base on ergonomics --a case study of lu jia xin wu [J]. Design, 2018 (1): 148-149.

[3] WANG Shengfei. Study on design strategy of rehabilitation garden [D]. Hangzhou: Zhejiang A\&F University. 2017.

[4] LIU Shuzhen, SUN Jianping. Ming su-style chairs design and human body engineering analysis [J]. Forest Engineering, 2014, 30 (1): 104-107.

[5] NI Haiyong, YAN Xiaoxing, PENG Wenwen, ZHONG Shilu. Analysis of bamboo furniture design based on human engineering [J]. Art Science and Technology, 2019 (9): 53.

[6] ZHANG Hongsheng. Research on human engineering of ming style furniture [J]. Art and Literature for the Masses, 2019, 451 (01): 118-119.

[7] REN Jiao. Application of ergonomics in the design of furniture [J]. Packaging Engineering. 2014, (18): 50-52.

[8] REN Pengyu. Application of human engineering in furniture layout of urban elderly people [J]. Science guide. 2015 (7): 152-153.

[9] LI Yan. Research on kitchen design of residential buildings for the elderly in northern China [D]. Beijing: Tsinghua University. 2006.

[10] HU Xianwen. Great hidden in the city: Discussion on villa courtyard design from the angle of human engineering [J]. Art Education Research. 2014 (2): 59-59.

[11] LUO Deyu. Application of human engineering in marketing design of outdoor furniture in City [J]. China Business \& Trade. 2014 (21): 42-43.

[12] YUN Junhong. Application of human engineering in campus
Landscape design [J]. Jiangxi Building Materials. 2014 (1): 197-197.

[13] LI Xin. Application of human engineering in architectural Design [J]. Architectural Engineering Technology and Besign, 2015 (13): 353

[14] YE Ping, BAI Di, WANG Xiaojin. Discussion on the relation between interior design and architectural design [J]. Modern Decoration (Theory), 2017 (1): 37-38.

[15] RAN Jiachen, LI Wanglin. The key points of the design of the elderly housing under the background of active aging [J]. Construction Materials \& Decoration, 2017 (2): 120-121.

[16] YANG Jianxin. Study on indoor behaviour pattern and spatial demand of nursing homes in northeast China [D]. Changchun: Jilin Jianzhu University, 2017.

[17] CHENG Kang, YIN Zhefang, WANG Zheng, WU Yani, WEN Yuhang. Effect of compound fragrance on physical and mental health status of female college students [J]. China Medical Herald, 2017, 14 (10): 48-51.

[18] Nikjou R, Kazemzadeh R, Asadzadeh F, Fathi R, Mostafazadeh F. The effect of lavender aromatherapy on the symptoms of menopause [J]. Journal of the National Medical Association, 2018, 110 (3): 265-269.

[19] JIA Dapeng WANG Xinjie LIU Yu. Forest Health Assessment of Jingouling Forest Farm [J]. Journal of Northeast Forestry University, 2019, 47 (08): 47-52+57.

[20] TANG Jianxiong, CHEN Ni ng, MA Mengyao, LI Youbei. The spatial structure and disparities of healthy tourism destinations in Changsha-Zhuzhou-Xiangtan [J]. Journal of Shaanxi Normal University (Natural science Edition), 2019, 47 (3): 115-124.

[21] GAO Xiang, YAO Lei. Preliminary Study on the Combinations of Specific Aromatic Plants for Hypotensive Healthcare [J]. Chinese Landscape Architecture, 2011, 27 (04): 37-38.

[22] JIN Hexian. The culture of Mei flower and Sweet-scented Osmanthus, analysis of volatile compounds during blooming season from these two flowers and their aroma' influence on human health [D]. Beijing: Beijing Forestry University, 2003.

[23] GAO Yan. VOCs release dynamics and their effects on human health in greening trees in Beijing [D]. Beijing: Beijing Forestry University, 2005.

[24] LU Qi, PENG Aiming, LIU Shuangxin, TU Wenjian, HU Kuijuan, GAO Jian. Influence of Chinese Daffodil Fragrance on Human Blood Pressure, Heart Rate and Respiratory Rate [J]. Journal of Anhui Agricultural Sciences. 2010, 38 (26): 14329-14330.

[25] LIAO Jianjun, QI Zengxiang, LI Tao, WANG Kuan. Research advances in volatile organic compounds of plant [J]. Journal of University of South China (Science and technology), 2016, 30 (3): 119-123.

[26] ZHAO Qing, QIAN Wanhui, TANG Honghui, YANG Qing, YAN Jun. Differences of health care functions of six forest stands in Yunyong forest park, Guangdong [J]. Journal of Zhejiang A\&F University, 2018, 35 (04): 750-756.

[27] LIANG Rui. Study on the effects of volatile organic compounds from aromatic plant Cinnamomum Burmann on human health in environmental spacec [D]. Kunming: Southwest Forestry University. 2019. 
[28] HU Huiming, ZHAO Chuzhi, ZHAO Zhaoyi, ZHANG Xin, RAN Linghua. Study on the correlation of Chinese adult body size data [J]. Chinese Journal of Ergonomics, 2014, 20 (3): 49-53.

[29] WU Dan. Talking about how to use ergonomics to improve seat comfort [J]. Charming China. 2012 (31): 72.

[30] ZHANG Hongping, SHE Zhijia, CHEN Hua, CHEN Weijie. Inclusive Design for Elderly People of Urban Park in Zhaoqing [J]. Guangdong Landscape Architecture, 2015 (6): 44-47.

[31] WANG Fengyu. Investigation on the use of the elderly in urban parks based on POE method [D]. Shenyang: Shenyang Agricultural University, 2016.
[32] HAO Zezhou WANG Cheng XU Xinhui WANG Yanying ZHANG Chang DUAN Wenjun WANG Ziyan JIN Yibo. Effect of Shenzhen Urban Forest Soundscape on Psychological and Physiological Changes of Human Beings [J]. Journal of Northwest Forestry University, 2019, 34 (3): 231-239.

[33] ZHANG Jing. Meditation training affecting the heart rate variability (HRV) and brain oscillations in middle and elderly women [J]. Shandong Sports Science \& Technology, 2018 (6): 66-70.

[34] KANG Ning, LI Shuhua, LI Fahong. Study on the effect of different landscapes on human psychology [J]. Chinese Landscape Architecture, 2008 (07): 69-72. 